\title{
Initial Flight Testing of an eXternal Vision System (XVS) for the Low Boom Flight Demonstrator (LBFD)
}

\author{
Lynda J. Kramer, ${ }^{1}$ Randall E. Bailey, ${ }^{2}$ Steven P. Williams, ${ }^{3}$ Trey Arthur, ${ }^{4}$ Kevin J. Shelton, ${ }^{5}$ Kurt Severance, ${ }^{6}$ and \\ Kemper S. Kibler, ${ }^{7}$ \\ NASA Langley Research Center, Hampton, VA, 23681-2199, USA
}

\begin{abstract}
NASA will design an eXternal Vision System (XVS) that, with other aircraft systems and subsystems, will ensure safe and efficient operations in all phases of flight for its Low Boom Flight Demonstrator vehicle. XVS is a combination of display, sensor, and computing technologies, creating an electronic means of forward visibility for the pilot. A flight test was performed evaluating a preliminary design of an XVS to quantify, by direct comparison, the ability of a pilot using an XVS to see and recognize airborne traffic compared to that of a pilot using forward-facing windows during challenging see-and-avoid scenarios. The data showed that the XVS and forward-facing windows were essentially equivalent in detecting and recognizing incurring traffic aircraft. The data also showed that the pilot using the XVS could see and recognize the incurring traffic at no less than $0.7 \mathrm{~nm}$ prior to the pilot using the forward-facing windows. The performance of the XVS was dependent upon the application of image contrast enhancement. Recommendations for future improvements were captured from evaluation pilot commentary.
\end{abstract}

\section{Introduction}

NASA's Low-Boom Flight Demonstration (LBFD) project is being developed to validate design tools and technologies applicable to low sonic boom aircraft. A critical part of this work is to create a database of community response supporting the development of a noise-based standard for supersonic overland flight. Under NASA's Aeronautics Research Mission Directorate, a set of LBFD aircraft capabilities has been defined to conduct effective community response studies. The LBFD objectives would be accomplished through the design, construction, and flight validation of a sub-scale research aircraft that creates a shaped sonic boom signature with a calculated loudness level of 75 PLdB (Perceived Level (PL), dB) or less during supersonic cruise (Mach $\geq 1.4$ ) flight. A NASA-led team will conduct low-boom community response overflight studies with multiple test campaigns using the LBFD aircraft over various locations across the continental United States. The campaign will develop a low-boom community response database that will be provided to the Federal Aviation Administration(FAA) and International Civil Aviation Organization (ICAO) Committee on Aviation Environmental Protection (CAEP) in support of their development of a noise-based standard for supersonic overland flight.

The overall configuration and forward fuselage / nose shaping of the LBFD design are critical to meeting the program goals for sonic boom signature. But this shaping significantly impacts the natural vision for the pilot provided by cockpit windows. Forward-facing windows are just not practical for this design.

NASA will design an eXternal Vision System (XVS) that, with other aircraft systems and subsystems, will ensure safe and efficient operations in all phases of flight. XVS is a combination of display, sensor, and computing technologies which create an electronic view, forward of the cockpit, analogous to forward-facing windows.

XVS is being designed to provide sufficient forward visibility so that the LBFD aircraft can be permitted timely, routine access to and operations within the National Air Space without the need or requirement for special handling by Air Navigation Services Providers (ANSPs), constraints to airspace classes, or chase aircraft-required support by

${ }^{1}$ Aerospace Engineer, Crew Systems and Aviation Operations Branch, Non-member.

2 Lead Aerospace Engineer, Crew Systems and Aviation Operations Branch, Non-member.

${ }^{3}$ Research Engineer, Crew Systems and Aviation Operations Branch, Non-member.

${ }^{4}$ Research Electronics Engineer, Crew Systems and Aviation Operations Branch, AIAA Senior member.

${ }^{5}$ Flight Test Engineer, Aircraft Operations and Engineering Branch, Non-member.

${ }^{6}$ Computer Engineer, Flight Software Systems Branch, MS 472, AIAA Associate Fellow

${ }^{7}$ Computer Engineer, Simulation Development and Analysis Branch, Non-member. 
providing an equivalent means of natural visibility for the pilot. The goal is that XVS, in addition to other LBFD systems, will allow the LBFD to be treated analogous to a "normal" piloted aircraft (i.e., to be able to "file and fly"). XVS is an electronic window, creating an electronic means of forward visibility for the pilot.

NASA has been actively researching vision systems technologies and XVS, in particular; hence a pedigree of experience and data are available. However, requirements by which an electronic means of vision/visibility - an XVS - can meet equivalent levels of performance and safety to forward-facing windows have not been developed and validated.

\section{Approach}

The LBFD XVS design approach employs off-the-shelf, near state-of-the-art equipment to provide forwardvisibility analogous or possibly, equivalent to windows and to promote safe and efficient LBFD operations in all phases of flight despite the absence of forward-facing windows. This approach provides an affordable, minimal risk path toward deployment in support of the LBFD vehicle.

The XVS team will also capture design, test, analysis, and flight data during this program to help create design standards for an electronic means of vision to provide equivalent performance and safety to forward-facing window. These works, as well as regulatory and operational approval lessons-learned, will be disseminated to industry and other government agencies for their knowledge and use as an XVS is envisioned as a necessary requirement for operational supersonic aircraft.

A series of test and evaluation campaigns will be used during development, with possible modifications and improvements being made as necessary and appropriate after these tests, to meet LBFD XVS goals. This paper describes the first flight campaign in this series.

\section{Background}

The precise requirements to meet the goal of equivalent performance and safety to forward-facing windows have not been quantified per se, but functional requirements are being identified from various sources.

Title 14 of the Code of Federal Regulations (14 CFR), including Part 25.773 and its associated Advisory Circular (AC) succinctly states that "the pilot compartment, its equipment, and its arrangement to include pilot view, must allow each pilot to perform his or her duties, including taxi, takeoff, climb, cruise, descent, approach, landing, and perform any maneuvers within the operating envelope of the airplane, without excessive concentration, skill, alertness, or fatigue.”

This objective is especially problematic for an XVS design during Visual Meteorological Conditions (VMC) when flight crew normally - with forward-facing windows - have sufficient natural visibility of the surrounding airspace and terrain (whether or not they may be operating under Visual Flight Rules (VFR) or Instrument Flight Rules (IFR)). Operations during these conditions create the driving XVS design standards - derived from the three tenets of VMC/VFR operations - which apply to all aircraft in these visibility states: "see-and-avoid", "see-to-follow", and "self-navigation".

"See-and-avoid" is the fundamental aviation tenet codified within Part 91, §91.113. See-and-avoid requires that visual vigilance, outside the aircraft, shall be maintained at all times, by each person operating an aircraft, regardless of whether the operation is conducted under IFR or VFR to avoid collision with other aircraft, vehicles, or the terrain and to remain "well-clear."

"See-to-follow" is an operational capability employed by ANSPs when natural vision conditions are sufficiently clear that, upon flight crew concurrence, directs an aircraft, for example, to follow another aircraft to a runway for landing or another aircraft or vehicle during surface operations. When "see-to-follow" authorization is given, the flight crew is now responsible for safe separation from that aircraft (including wake separation), the terrain, and obstacles and ANSP guidance for navigation to the destination is no longer given.

"Self-navigation" is an operational concept that, when natural vision conditions are sufficiently clear, allows the pilot to visually navigate to a destination and recognize significant navigational references. "Self-navigation" includes the use of specific charted procedures such as the Washington National Airport River Visual to Runway 19 and the Parkway Visual arrival to John F. Kennedy Airport Runways 13L/R. "Self-navigation" is also a regulatory requirement. This includes a pilot's required recognition and identification of specific visual references to enable descent belowthe published minimum descent altitude, decision height, or decisionaltitude on an instrument approach procedure (e.g., see 14 CFR 91.175) and the pilot's ability to control the aircraft descent rate, control and maintain the aircraft's trajectory toward the intended touchdown point while remaining within the visual segment of the approach path, and achieve a touchdown with acceptable descent rate within the intended touchdown zone and without incurring

a tail or empennage strike on the runway. Finally, "self-navigation" also includes, for example, surface operations 
where the pilot must recognize all signs, lights, and painted surfaces and identifying and adhering to their intent (e.g., hold short lines, "spot markers").

\section{See-and-Avoid XVS Design Objective}

Ground simulation, analysis, and flight testing data suggest that the see-and-avoid requirement creates the driving, critical design constraints (Ref. 1).

These data also show that it is difficult, if not impossible, to adequately and completely specify equipment and technology requirements from see-and-avoid functional requirements because the ability of a human observer to identify and recognize other aircraft and vehicles is dependent upon an almost infinite combination of variables in the real-world, not all related to the vision systems technologies. The variables include, for example, the characteristics of the traffic aircraft including its size, color, and orientation [Ref. 2, 3]. The variables also include the background of the scene, the illumination of the traffic aircraft, and the atmospheric conditions through which it is being viewed.

Ideally, the ability of an XVS to demonstrate equivalent see-and-avoid performance and safety would be met by flying an XVS-equipped research aircraft against different sized, traffic aircraft in multiple traffic scenarios and compare the performance of an observer using an XVS to that of the same observer looking through forward-facing windows at identical times and conditions.

Meeting this objective exactly is, of course, impossible. Instead, an innovative test method was employed using side-by-side, see-and-avoid comparison between XVS and forward-facing windows, albeit with different, but equally qualified observers.

The test objective was accomplished using a modified between-subjects test design with two evaluation pilots (EPs) on-board a UC-12 test aircraft. One EP was seated in the cockpit, using the forward-facing windows, and the other EP, located in the cabin, using the XVS. The EPs would rotate positions during the test flight.

\section{Research Equipment}

Our testing used a NASA Langley Research Center (LaRC) UC-12 aircraft (Figure 1). Research equipment installed on the test aircraft included: two cameras (one Ultra-High Definition(UHD) visible spectrum camera and one standard definition (SD) visible spectrum camera); a research pallet; a UHD monitor; XVS processing computer; and aircraft instrumentation and data bus interfaces for aircraft state data and Autonomous Dependent SurveillanceBroadcast (ADS-B) traffic data. The UHD and SD cameras, each with a fixed forward field-of-view (FOV), served as the XVS sensors and were mounted behind the windscreen (Figure 2). (The SD camera was used for comparative demonstrations of performance against the UHD camera. These data are not reported herein.) Both cameras were mounted to look along the longitudinal axis of the plane with as little pitch, roll, or yaw as possible (i.e., nominally 0 degrees). Additionally, both cameras were mounted so that any UC-12 fuselage blocking of their respective FOVs was minimized.

The UHD camera was an IO Industries Flare 12M180CCX-M color UHD camera. The camera has 3920 x $2400-$ pixel resolution with a Bayer color format. The camera lens was a 35mm fixed focal length lens (Canon EF 35mm f/1.4L II USM L-Series Lens).

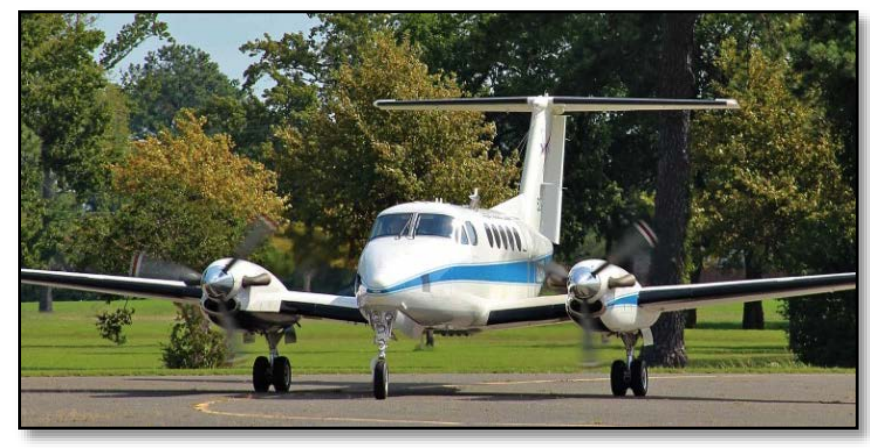

Fig. 1 UC-12 Test Aircraft 


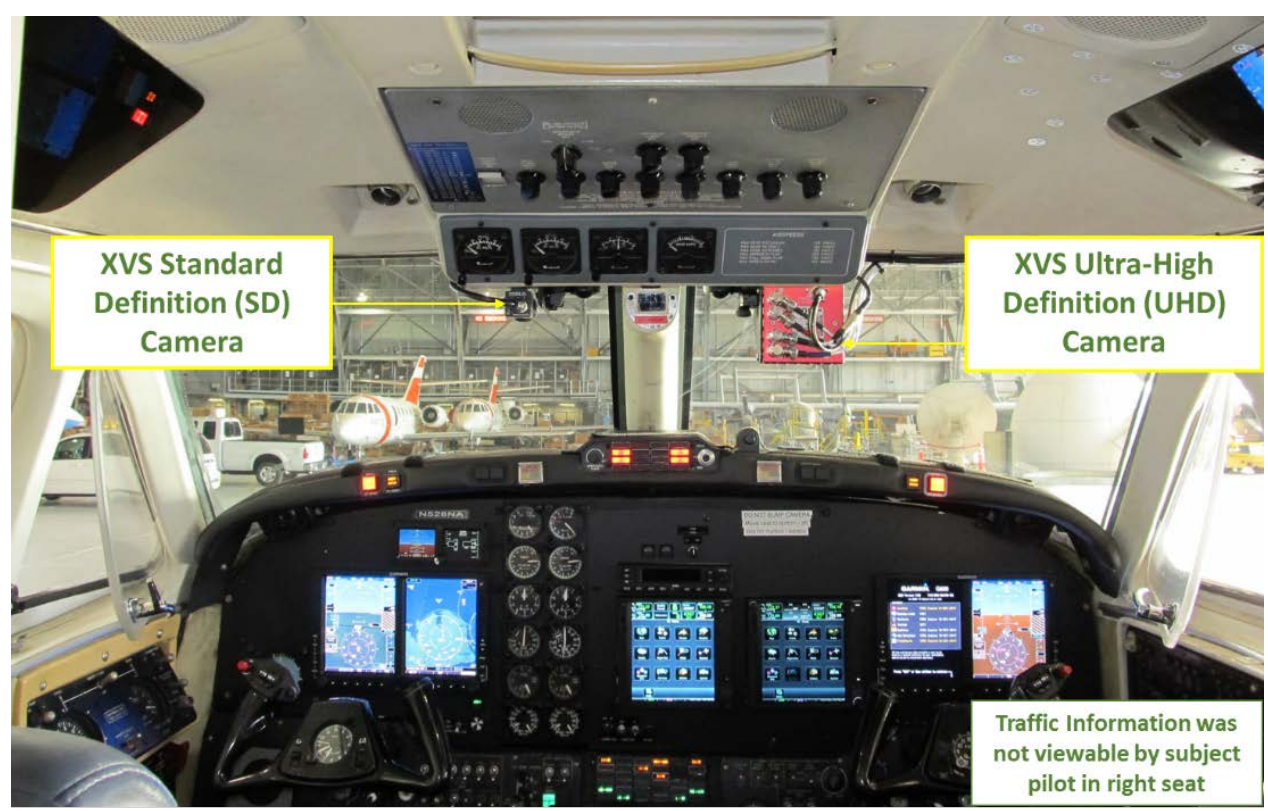

Fig. 2 XVS Cameras mounted in Forward Window

The XVS UHD monitor was a Viewpoint Systems VPT-24UHD-MIL monitor mounted in the UC-12 cabin (see Figure 3). The monitor is 22.5 inches wide $x 14.8$ inches high $\times 3.2$ inches deep in size.

The FOV of the UHD monitor, when viewed from the design eye reference point-a nominal distance of 35 inches from the monitor - subtends 36 deg Horizontal by 24 deg Vertical. The FOV of the UHD camera and the FOV of the UHD monitor subtend essentially the identical area, thus, providing a conformal display for the observer.

The UHD camera imagery was fed to a Trenton Systems THS2085 HDEC 2U rack-mounted computer using a CoaXPress interface to a Matrox Radient eV-CXP video capture card.

This computing system has two (2) 10 Giga-Byte (GB) Ethernet interfaces and two (2) 1 GB Ethernet interfaces directly on the mother board, slots for the graphics subsystem, the Quad CoaXPress interface subsystem, and the SVGA (for the SD camera) interface subsystem. The mother board has two 14 core $2.3 \mathrm{GHZ}$ processors (total of 28 CPU cores), 32GB of system memory, and a 2 Terra-Byte solid state hard drive. The computer also used an NVidia GTX 1070 graphics card with $19201.5 \mathrm{GHz}$ cores and 8 Gigabytes of memory. The computer fits a standard 18 inch deep $2 \mathrm{U}$ rack and weighs approximately 22 lbs.

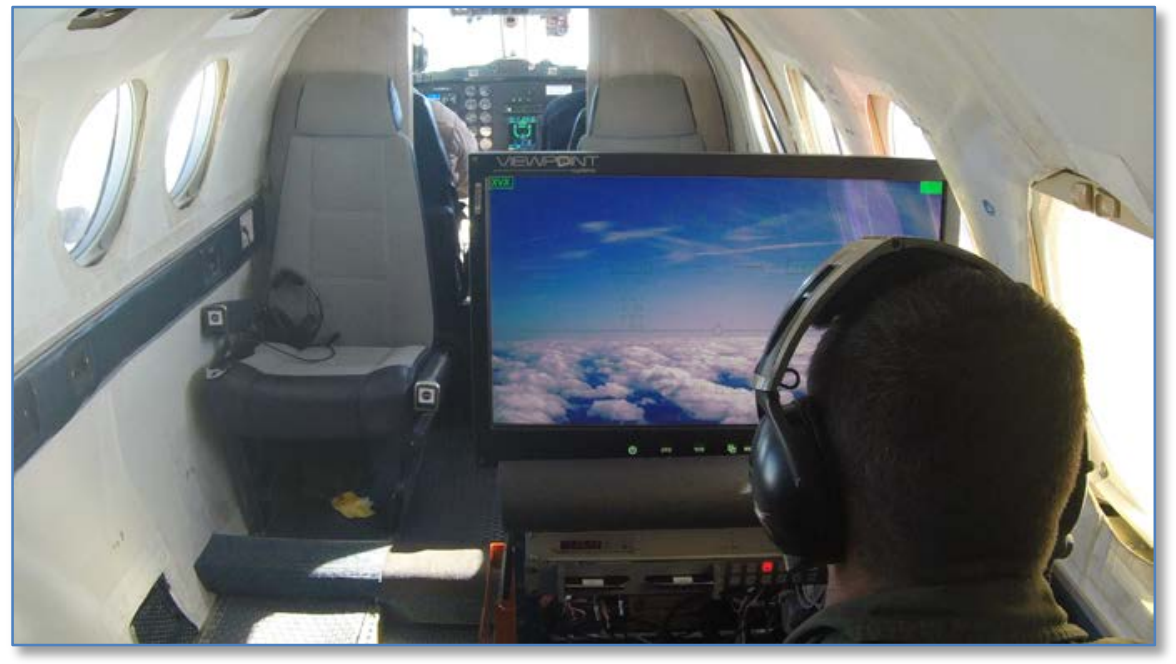

Fig. 3 Over-the-shoulder view of XVS Monitor installation in Cabin 
For this initial flight test, the Bayer format imagery was converted to Red-Green-Blue (RGB) for image processing using a simple symmetric bilinear interpolation. At each pixel of the image, this process uses the color value of the pixel and interpolates to get the other two colors (in color space) at that pixel from its nearest neighbors. RGB was then converted by Hue, Saturation, and Luminance (HSL) for image contrast enhancement, if applied.

The Contrast-Limited Adaptive Histogram Equalization (CLAHE) algorithm (Ref. 4) was varied experimentally for image contrast enhancement on the luminance channel.

The UHD monitor served as the XVS display and had independent imagery and symbology brightness control for the EP.

The XVS principal investigator (PI) used a touchscreen tablet as a small, portable interface to configure the XVS display software modes for experiment data runs.

\section{Research Goal}

The research goal was to quantify, by direct comparison, the ability of a pilot using an XVS to see and recognize airborne traffic compared that of a pilot using forward-facing windows during challenging see-and-avoid scenarios.

\section{Test Procedure}

During each flight, one pilot was seated (identified as Subject 1, in Figure 4) in the cockpit right seat and another pilot (Subject 2 in Figure 4) was seated at the XVS display in the cabin (Figure 3).

See-and-avoid traffic scenarios were flown with the evaluation crew being observers. A total of 12 see-and-avoid scenarios were run each flight. The experiment design switched the subject seating location (Subject 1 in the cabin at the XVS display and Subject 2 in the right seat of the cockpit) midway through the flight. Each subject crew flew two flights.

The see-and-avoid scenarios were various combinations of encounters that emphasized an expanding traffic image; that is, the traffic was generally at the same azimuth and elevation angle in the subject's field-of-regard but grew in size (subtended visual angle) as it got closer to the test aircraft. This maneuver is in contrast to one where the traffic aircraft translated or moved across the field-of-regard (i.e., the traffic included movement in azimuth and/or elevation angle in the subject's field-of-regard). Two-sized traffic aircraft were used (Figure 5): a small-size aircraft (Lancair LC-40) and a medium-sized aircraft (Falcon Jet HU-25).

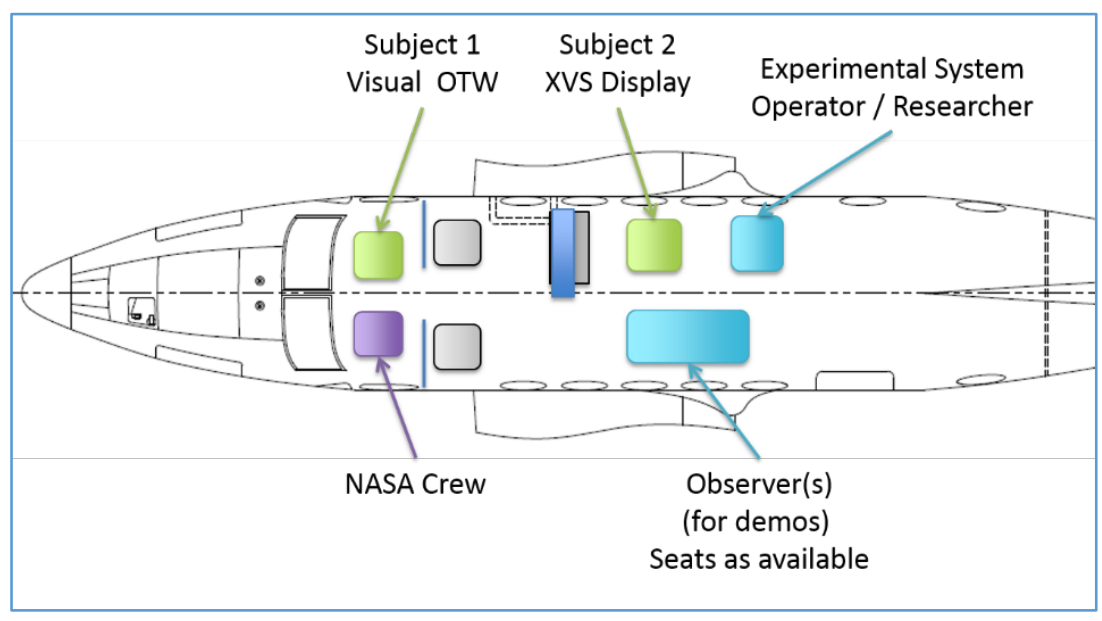

Fig. 4 XVS Crew Arrangement in Be-200 aircraft 

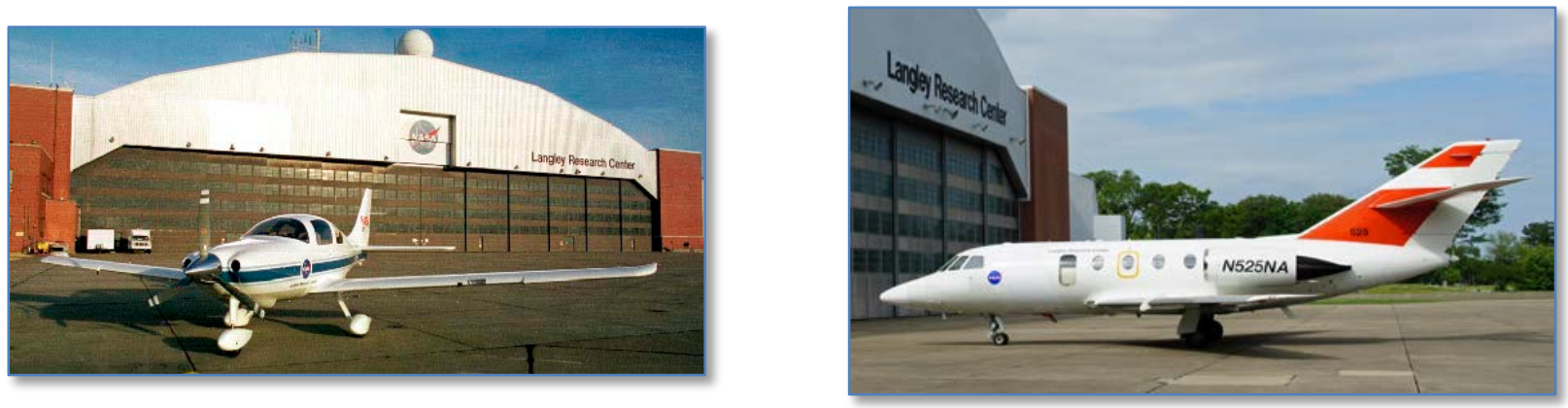

Fig. 5 Target aircraft - Small (left) Lancair LC-40 and Medium-sized (right) Falcon Jet (HU-25)

The see-and-avoid traffic scenarios are illustrated in Figures 6 and 7. For safety-of-flight, the traffic was separated horizontally and/or vertically from the test aircraft. Numerous other additional mission rules were in effect to ensure safety-of-flight.

In Figure 6, the co-altitude head-to-headscenario is shown. The horizontal separation was nominally $500 \mathrm{ft}$. Long, straight, prominent ground features (e.g., railroad tracks) were used in addition to Global Positioning System navigation and waypoints to ensure separation.

The head-to-head scenario was also flown with no vertical separation (i.e., co-altitude) or with $+/-500 \mathrm{ft}$ altitude. These six maneuvers provided a variety of encounter geometries with all being expanding traffic encounters (at least, until the two aircraft were in close proximity).

The other scenario was a climbing head-on encounter (shown in Figure 7). In this maneuver, the ascent of the traffic aircraft was managed to maintain a relatively constant intercept angle as it climbed out from the ground clutter. (The traffic aircraft leveled off at $500 \mathrm{ft}$ lower than the test aircraft to ensure separation.)

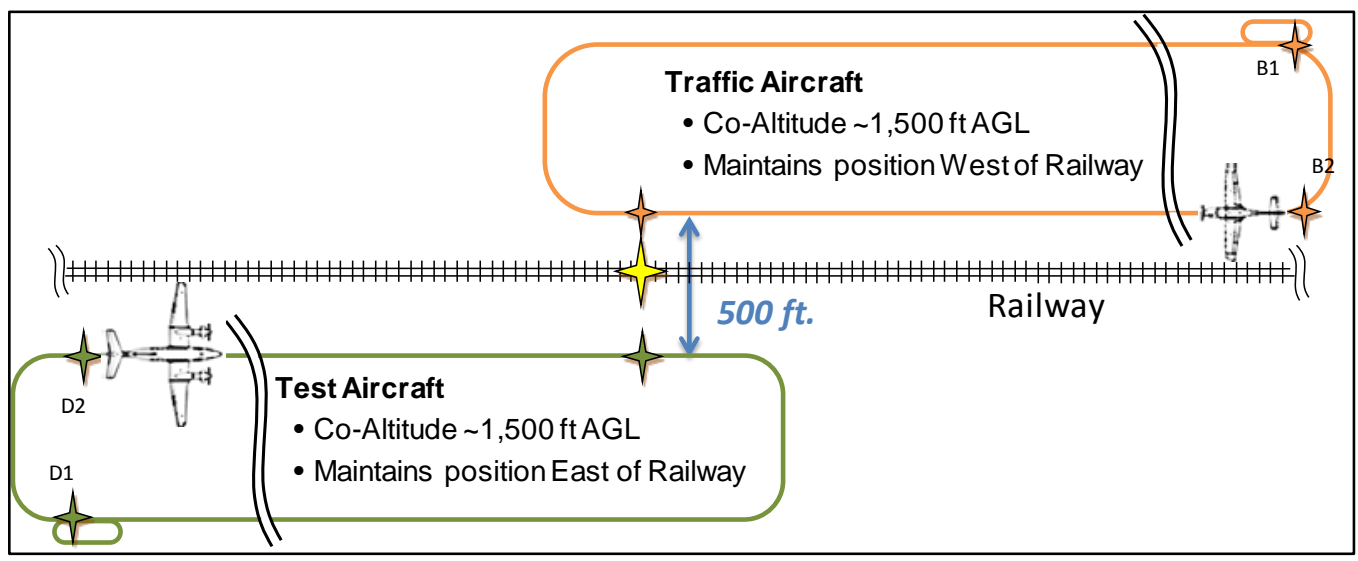

Fig. 6 Head-to-Head Co-Altitude Scenarios (Top-Down View)

Once the test and traffic aircraft were in position to begin a run, the subjects were told to begin their scan for traffic. The subjects were not told which encounters were being run.

Two push-button pilot event markers, one in the flight deck for Subject Pilot 1 and one in the cabin for Subject Pilot 2, were provided. The push button event markers were utilized by the two EPs to identify when the target aircraft was seen, either visually out-the-window or on the XVS display. The subjects were told to point to (and not verbalize) the traffic location. This process was used to verify the accuracy of their detection. Once both subjects saw the traffic aircraft or the aircraft passed each other, the safety pilot called 'knock it off' and the run ended.

In total, eight data collection flights were flown against the two different sized participating traffic aircraft for a nominal total of 96 see-and-avoid traffic runs. The test generated XVS performance data, including comparisons to natural vision. Post-run subjective acceptability data were also collected.

The primary evaluation measures were the time and range for traffic detection and recognition by two human observers, one visually out the forward window and one with the XVS. 


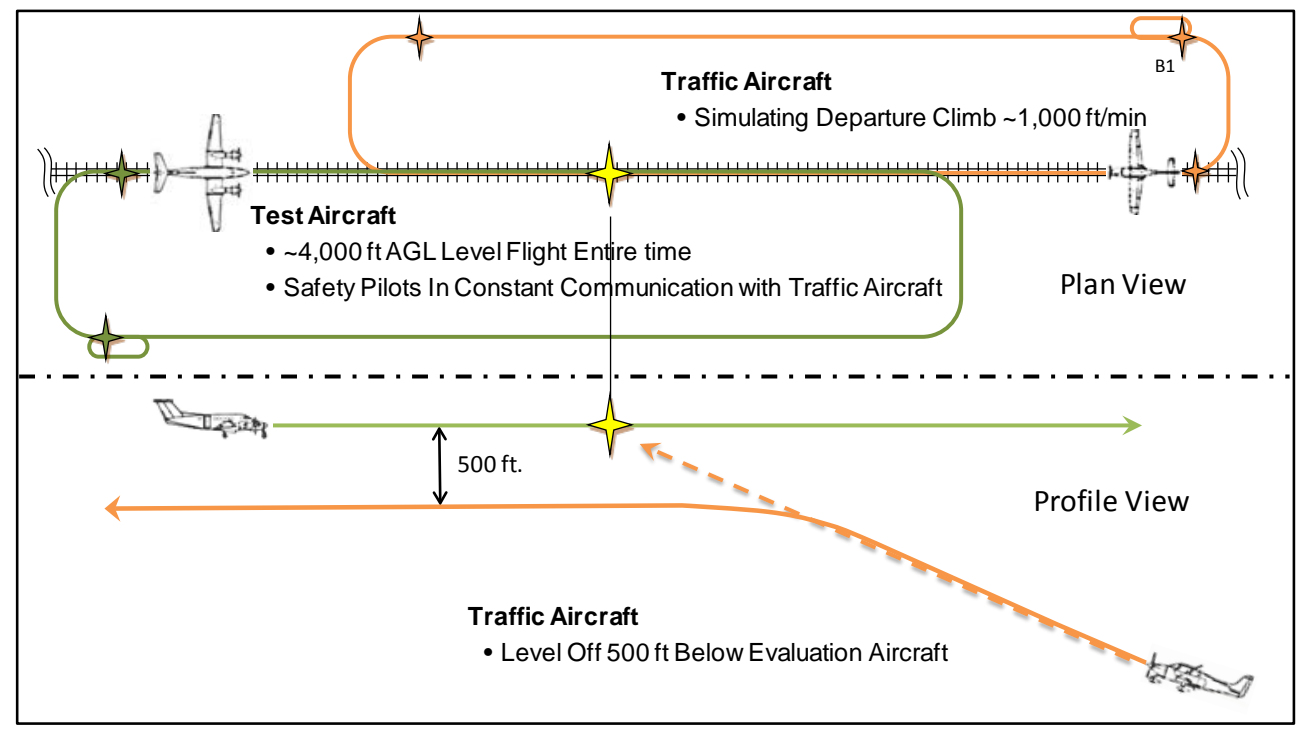

Fig. 7 Head-to-Head Climbing Scenario (Top Down and Side View)

\section{Research Objectives}

Within the stated research objective, the flight test experiment explored the effects of aircraft size (small and medium) and the see-and-avoid scenario. For data analysis, we have collapsed the scenarios into one of two: headto-head, constant altitude and head-to-head climbing. The constant altitude head-to-head maneuvers, whether coaltitude with lateral separation or $500 \mathrm{ft}$ above or below with vertical and lateral separation, were essentially the same task. The traffic aircraft did not markedly translate across the field-of-regard (i.e., it was only when the traffic aircraft got within a mile or so of the test aircraft that the horizontal or altitude differences became notable) and was masked in the background of the horizon.

The other manipulation in this test was the influence of using raw, or unprocessed, UHD camera imagery or contrast-enhanced UHD imagery for the XVS (Figure 8). Previous XVS flight tests (Ref. 5) have shown that resolution is one determinate of traffic visual acquisition performance, but image contrast can be as important, if not more so. Traffic detection, among many other factors, is determined by the relative contrast between the traffic and the background in which the traffic is viewed.

Numerous image contrast algorithms have been developed and tested. Interestingly, most systems for traffic detection focus on InfraRed (IR) sensor imagery. Most image contrast algorithms that use color or visual imagery are aimed at realistic or artistic portrayals (i.e., photography, entertainment). In this flight test, the CLAHE algorithm was used (Ref. 4).

Image contrast enhancement by using multiple spatial windows for image enhancement has been identified in previous tests (Ref. 5) as being a critical feature since the luminance differences between the sky and ground can be extreme. The CLAHE algorithm segmented each UHD image into 576 regions ( 24 x 24 array) smaller regions with each region containing 160 x 90 pixels. In each of these regions, the luminance histogram of the output was transformed to approximately match a uniform distribution. The small transformed image regions are then combined using bilinear interpolation to minimize artificially induced boundaries in the transformed UHD output. An example of the image contrast enhancement is shown in Figure 8.

A trade study is on-going for XVS contrast enhancement on the LBFD XVS implementation which compares and contrasts performance and real-time computational burdens. The results of this test and others will be used for final algorithm selection. 

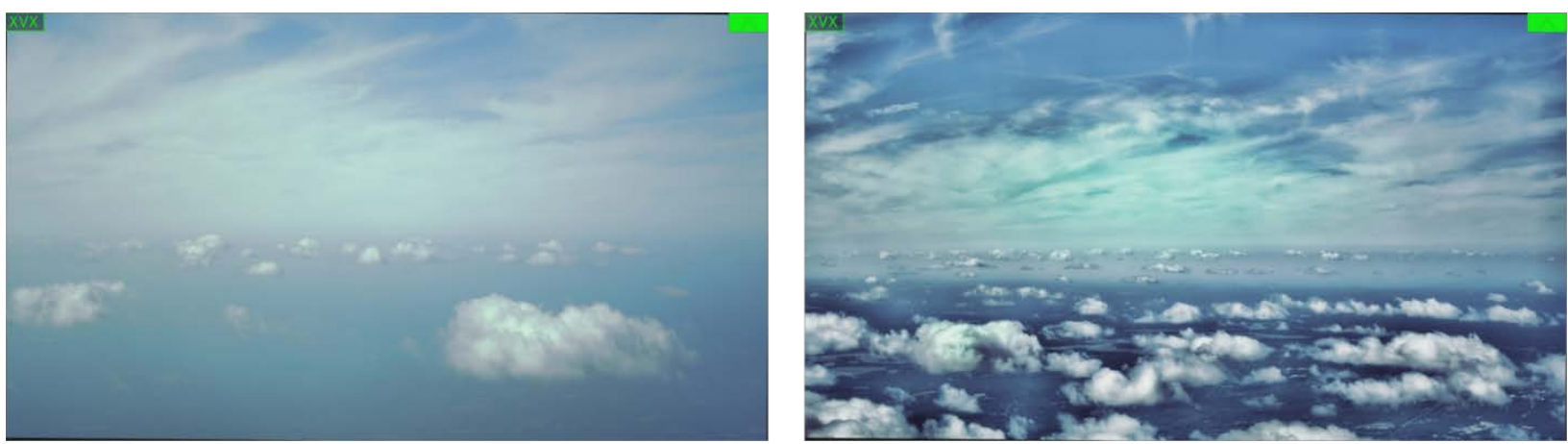

Fig. 8 XVS with unprocessed imagery (left picture) and XVS with processed imagery (right picture)

\section{XVS Flight Test Summary}

Eight pilots, paired into four subject crews, were test subjects. All pilots were former military pilots and they had an average of over 4,700 hours flight time in slightly over 25 years of flying. All pilots had 20/20 corrected or uncorrected vision. None of the pilots had a color deficiency.

Approximately 30 hrs of flight time on the UC-12 aircraft were expended for data collection.

The tests were conducted in the local area of Langley Research Center in September and October 2017. The flights were conducted in VMC with visible ground references. The estimated flight visibility was between 10 and 25 miles for all flights. The flights were flown on occasion with scattered cloud layers below and scattered to overcast layers above. As can be expected in Virginia, haze was a prevalent atmospheric condition.

Each crew flew at least 12 runs with each sized aircraft. Four extra runs were also flown as repeat data points for a total of 100 traffic detection runs. The distribution of these runs are shown in Table 1 . The table shows that the runs were equally distributed between the variables of target aircraft size and XVS Image Processing (IP) on and off.

Table 1 Number of Runs

\begin{tabular}{|l|c|c|}
\hline & IP-On & IP-Off \\
\hline \hline Small-sized Traffic Aircraft & 26 & 24 \\
\hline Medium-sized Traffic Aircraft & 25 & 25 \\
\hline
\end{tabular}

The distribution of specific scenarios flown are given in Table 2. In this table, the head-on runs are broken out by their horizontal and vertical offsets. The distribution between the horizontal and vertical offsets were not controlled per se. The runs were varied to minimize learning effects by the crew and also for operational convenience and efficiency.

For the remainder of this discussion, the constant altitude runs are assumed to be identical. In each of these, the traffic aircraft emerged from the horizon and was visually cluttered or obscured by cloud layers or haze effects. Approximately 17 runs were flown against each aircraft size (small or medium) and with each XVS IP condition (on or off)

Also shown in Table 2, eight climbing traffic runs were flown against each aircraft size (small or medium) and with each XVS IP condition (on or off). For the climbing scenario, the traffic aircraft were not visually cluttered by horizon obscurants (e.g., cloud layers and haze) but were masked by ground clutter. 
Table 2 Number of Runs Split by Scenario

\begin{tabular}{|c|c|c|c|c|c|c|c|c|c|}
\hline \multirow[b]{3}{*}{$\begin{array}{l}\text { Aircraft } \\
\text { Size }\end{array}$} & \multirow[b]{3}{*}{$\begin{array}{l}\text { IP } \\
\text { On/Off }\end{array}$} & \multicolumn{7}{|c|}{ Head-On Scenarios } & \multirow{3}{*}{$\begin{array}{c}\text { Climbing } \\
\text { Scenarios } \\
\text { Climbing } \\
\text { Total }\end{array}$} \\
\hline & & Co-Alt. & Co-Alt. & Low & Low & High & High & & \\
\hline & & Right & Left & Right & Left & Right & Left & $\begin{array}{c}\text { Head-on } \\
\text { Total }\end{array}$ & \\
\hline Medium & IP & 1 & 7 & 2 & 3 & 1 & 3 & 17 & 8 \\
\hline Medium & IP Off & 7 & 2 & 3 & 1 & 3 & 1 & 17 & 8 \\
\hline Small & IP & 8 & 1 & 3 & 2 & 2 & 2 & 18 & 8 \\
\hline Small & IP Off & 1 & 7 & 2 & 2 & 2 & 2 & 16 & 8 \\
\hline
\end{tabular}

\section{Traffic Aircraft Detection}

To successfully accomplish see-and-avoid, the pilot must first see and recognize traffic aircraft.

The data from this test showed that the pilot, using forward-facing windows (OTW) saw the traffic aircraft on 94 out of 100 runs. On six runs, the OTW pilot did not see the traffic.

Analysis of the OTW detections showed that on 13 of the successful detections, the traffic aircraft was outside of the FOV of the XVS. For fair comparison to the XVS and its limited FOV, these twelve detections are not considered to be 'successful'. The FOV for the OTW pilot was not limited in any way; the pilot could use the entire viewing area provided by forward-facing windows even though they were briefed that the traffic encounters were going to come from the forward viewing quadrant. The 12 encounters outside of the FOV of the XVS occurred when the traffic aircraft was very close to ownship and thus, at large azimuth and elevation angles to the test aircraft. For this reason, we are not counting these runs as being successful traffic aircraft recognitions. Our data analysis segment for aircraft detection began when the test and traffic aircraft were in position and the Pilot-in-Command called start of run and ended when the traffic aircraft was no longer visible within the FOV of the XVS display in either the OTW or XVS condition.

In Table 3, the traffic detection rates are shown. The tabular data shows the following:

- The overall performance using the XVS were essentially the same as or equivalent to the OTW. The overall rate was $81 \%$.

- Detection rate for different sized target aircraft was essentially equivalent. The detection rate for mediumsized target aircraft was $96 \%$; the detection rate drops to $66 \%$ for the small-sized target aircraft. This trend makes intuitive sense; the smaller the traffic, the more difficult it is to visually see and recognize the incursion.

- The use of image processing for XVS does not influence the detection rate for medium-sized aircraft but it does notably improve detection for small traffic aircraft. The detection rate goes up to $76 \%$ with image processing compared to only $56 \%$ without it.

Of the 19 encounters for which the pilot at the XVS could not see the traffic, nine of the runs were climbing traffic encounters and 10 constant altitude encounters. Although the numbers are essentially equal, the statistics highlight the difficulty of detection of traffic climbing toward the test aircraft with ground clutter in the background since only 32 climbing scenarios, compared to 68 constant altitude scenarios, were flown. This is a detection rate of $71 \%$ versus $85 \%$ for constant altitude encounters.

Of the 19 encounters for which the pilot at the OTW position could not see the traffic (i.e., ignoring the 12 late recognitions outside the XVS FOV), seven of the runs were climbing traffic encounters and 12 constant altitude encounters. For the OTW condition, the detection rates were less biased; the difficulty of detection of traffic climbing toward the test aircraft with ground clutter in the background was almost equally as challenging as the constant altitude encounters. The detection rates were $78 \%$ for the climbing scenario versus $82 \%$ for head-on encounters. 
Table 3 Traffic Detection Rate by Viewing Mode, Traffic Aircraft Size, and XVS IP

\begin{tabular}{|c|c|c|c|c|c|}
\hline \multicolumn{4}{|c|}{ XVS } & \multicolumn{2}{|c|}{ OTW } \\
\hline \multicolumn{4}{|c|}{$81 \%$} & \multicolumn{2}{|c|}{$81 \%$} \\
\hline \multicolumn{6}{|c|}{ (IP Off: $76 \%$ and IP On: $86 \%$ ) } \\
\hline \multicolumn{2}{|c|}{ Small } & \multicolumn{2}{|c|}{ Medium } & \multirow{3}{*}{$\begin{array}{c}\text { Small } \\
66 \%\end{array}$} & \multirow{2}{*}{$\begin{array}{c}\text { Medium } \\
96 \%\end{array}$} \\
\hline \multicolumn{2}{|c|}{$66 \%$} & \multicolumn{2}{|c|}{$96 \%$} & & \\
\hline IP Off & IP On & IP Off & IPOn & & \\
\hline $56 \%$ & $76 \%$ & $96 \%$ & $96 \%$ & & \\
\hline
\end{tabular}

\section{Traffic Aircraft Detection Margin}

To successfully accomplish see-and-avoid, the pilot must see and recognize traffic aircraft with sufficient time and distance to react and maneuver the aircraft to avoid collision.

Our data analysis segment for aircraft detection began when the test and traffic aircraft were in position and the Pilot-in-Command called start of run and ended when the traffic aircraft was no longer visible within the FOV of the XVS display.

The traffic aircraft transmitted their position using ADS-B and this information was received by the test aircraft. When the subject pilot pressed their event markers to indicate their detection and recognition of the traffic aircraft, the distance (range) between the two aircraft was recorded.

The range between the two aircraft when detected is shown in Figure 9. The data shows the range for the OTW and XVS detections using a box plot format. The box plot shows the median value by red horizontal line and the 25th and 75th percentiles indicated by the vertical and horizontal extent of the box. The notch in the box plot provides an estimate of the $5 \%$ significance level (i.e., the notch extremes correspond to q2 $\pm 1.57(q 3-q 1) / s q r t(n)$, where q2 is the median (50th percentile), q1 and q3 are the 25th and 75th percentiles, and $\mathrm{n}$ is the number of samples). Lastly, the whiskers show the minimum and maximum values within 1.5 times the interquartile range and red " + " symbols are identified as statistical outliers that extend beyond the range of the whiskers.

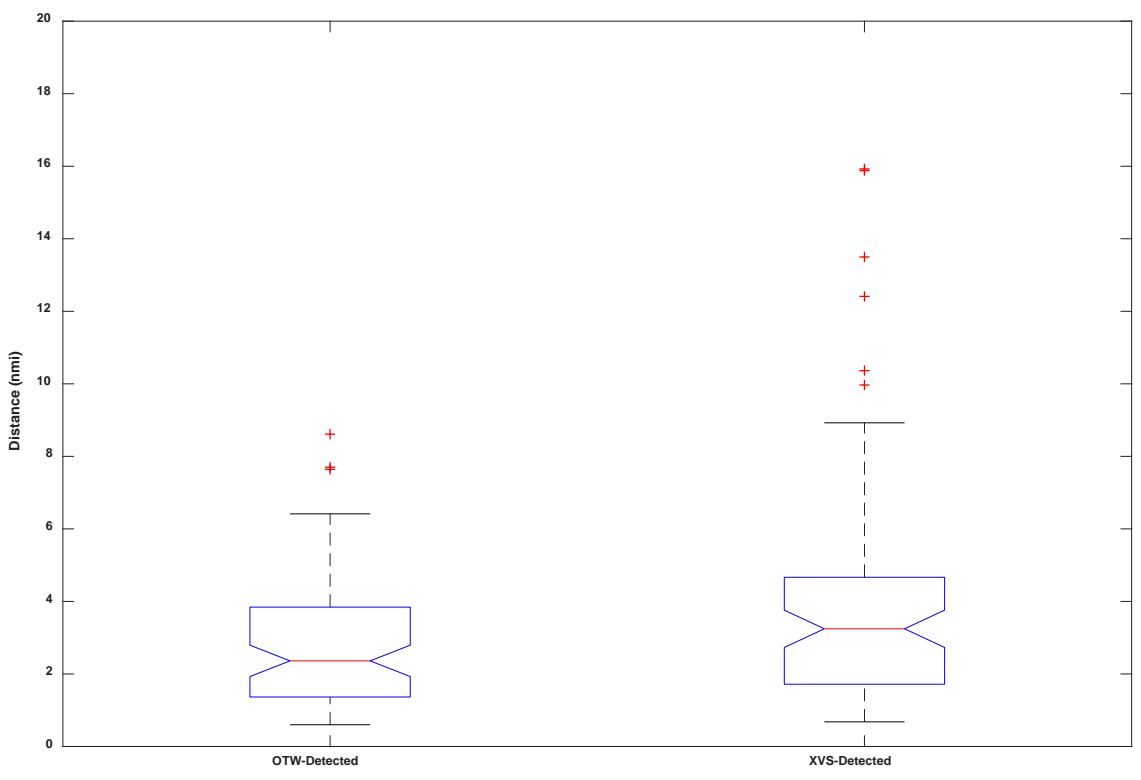

Fig. 9 Distance When Traffic Detected, Comparing XVS vs. OTW

The data show that the median distance for XVS detection was $3.2 \mathrm{nmi}$ compared to $2.4 \mathrm{nmi}$ for the OTW detections. The data suggests that the XVS performance is significantlybetter than OTW to the $5 \%$ significance level. The XVS pilot was able to detect the traffic aircraft sooner than the OTW pilot. The average closure rate for all traffic encounters was 380 knots, meaning that, half the time, the XVS pilot would have had 7.6 seconds more time to avert a collision. 
These data are broken out comparing the effect of traffic aircraft size in Figure 10. These data show that traffic size has a significant effect to the $5 \%$ level. The medium-size traffic was detected OTW, on average, at $3.3 \mathrm{~nm}$ from ownship, while the small-sized aircraft was only detected at $1.1 \mathrm{~nm}$. Similarly, the medium-size traffic was detected in the XVS, on average, at $3.9 \mathrm{~nm}$ from ownship, while the small-sized aircraft was only detected at $1.8 \mathrm{~nm}$.

These data also show that the performance for seeing and recognizing the medium-sized aircraft using XVS was better than using OTW (but not statistically significant). With an average closure rate of 420 knots, this difference of $0.6 \mathrm{~nm}$ during medium-sized traffic encounters equates to 5.1 seconds more time to avert a collision.

Similarly, the performance of seeing and recognizing the small-sized aircraft using XVS showed a trend towards being better than OTW but not significantly better. XVS had a median $0.7 \mathrm{~nm}$ improvement over the OTW observations. With an average closure rate of $318 \mathrm{knots}$, this difference of $0.7 \mathrm{~nm}$ during small-sized traffic encounters, equates to eight seconds more time to avert a collision.

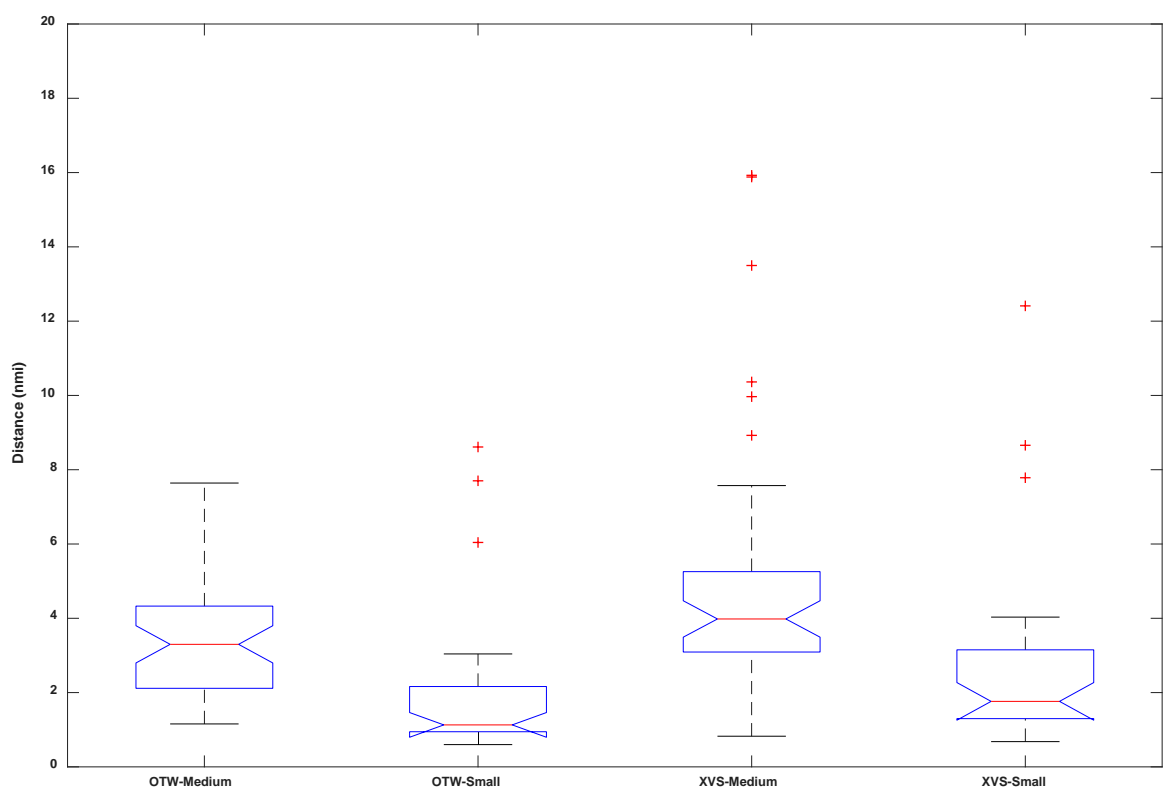

Fig. 10 Distance of Traffic when Detected; by Viewing Mode

The performance of XVS observations - that is, the detection range - is analyzed as a function of XVS image processing on/off. These data are shown in Figure 11 as a function of traffic aircraft size.

These box plot data show that image processing improved traffic detection. With image processing on, the medium-sized aircraft would be detected (median) at $5 \mathrm{~nm}$ from ownship and the small-sized traffic at $2.4 \mathrm{~nm}$ (median). Without image processing, the median detection of the medium-sized aircraft was down to $3.4 \mathrm{~nm}$ from ownship and the small-sized traffic at only $1.4 \mathrm{~nm}$ (median). 


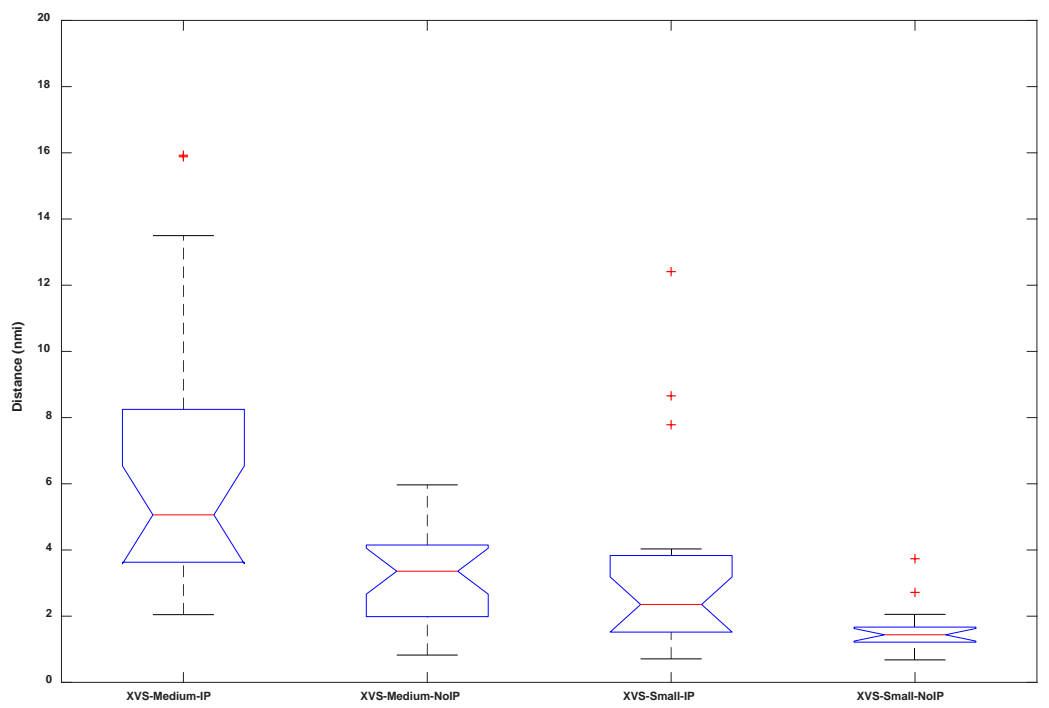

Fig. 11 Changes in Traffic Detection Range using XVS, Traffic Size and Image Processing (IP) On/Off

\section{Qualitative Traffic Aircraft Detection Performance}

Following the completion of each run, the pilots were asked to rate, using the Likert-type scale in Figure 12a, "the ease of detecting the traffic" and the "the ease in identifying the traffic". After these two questions they were also asked using the Likert-type scale (Figure 12b) to assess how strongly they agreed or disagreed with the statement "I had sufficient time to assess and react to the traffic I detected".

a) Ease of Detecting and Identifying Traffic

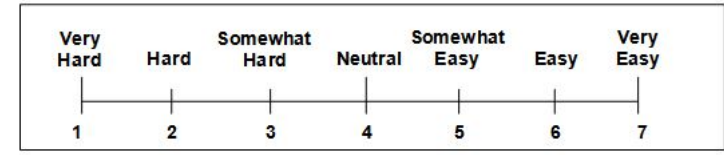

b) Sufficient Time To Assess And React To Traffic

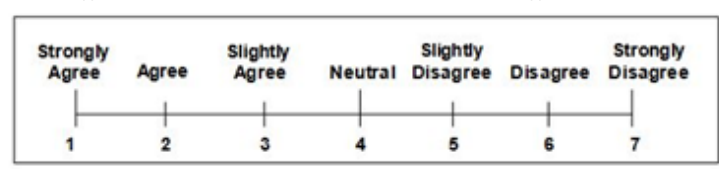

Fig. 12 Likert-type Scales for Quantitative Data

The post-run qualitative data for Question 1a - the ease of detecting the traffic - is shown in Figure 13 and the data for Question $1 \mathrm{~b}$ - the ease of identifying the traffic - is shown in Figure 14. The data highlights several points inherent to the test and the data.

- $\quad$ First - the ability of a pilot to see traffic and recognize it is a difficult task. (See Ref 2-3). It is a regulatory and safety-of-flight necessity inherent to the aviation, but it is nonetheless a visual perception challenge and creates a pilot workload burden. As such, there are relatively few ratings of "neutral" -4 - shown in Figures 13 and 14, for either XVS or OTW. The conditions (atmospheric and traffic size) created favorable or unfavorable results. As one pilot noted: "under the test and test conditions flown (day, hazy, below clouds) although OTW was better than XVS display, safe see-and-avoid functions with OTW was difficult for targets not well above the horizon (which was a clear blue background)" "environmentals greatly affect detection range.”

- In general, the pilots felt that XVS was either more difficult than OTW (see "very hard" ratings of 1, in Figures 13 and 14) or XVS tended to be easier (see "very easy" of 7, in Figures 13 and 14 compared to OTW). This dichotomy is also shown in Figure 15 for the response to Question 2 - sufficient time to react to traffic. More ratings of 1 ("strongly agree") were given for XVS than for OTW, but conversely, more ratings of 
"disagree” and "strongly disagree" (rating of 6 and 7, respectively) were also given to XVS compared to OTW.

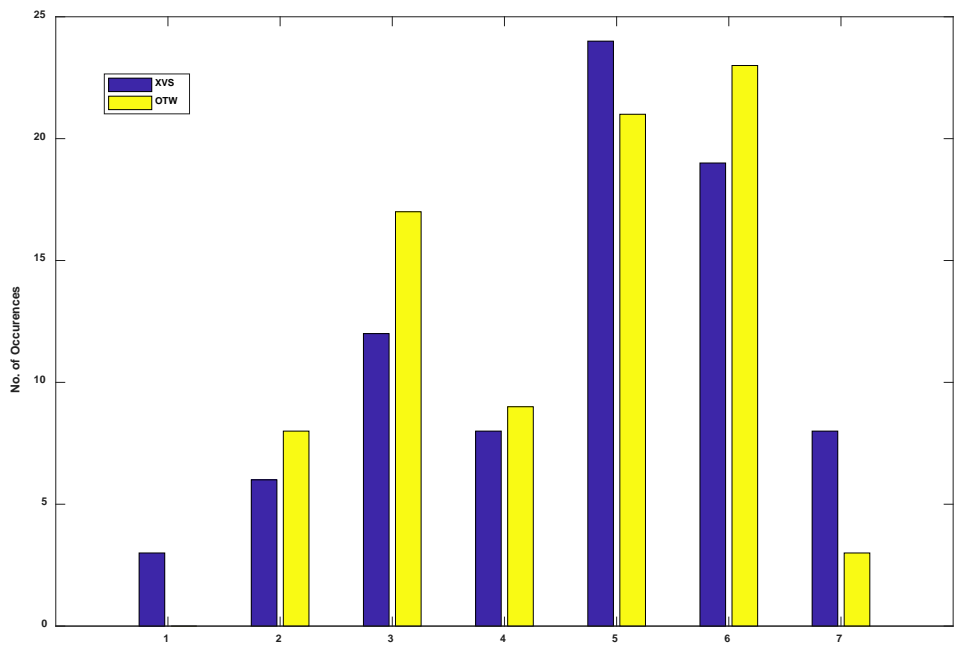

Fig. 13 Ease of Detecting Traffic Aircraft (1: Very Hard; 4: Neutral; 7: Very Easy)

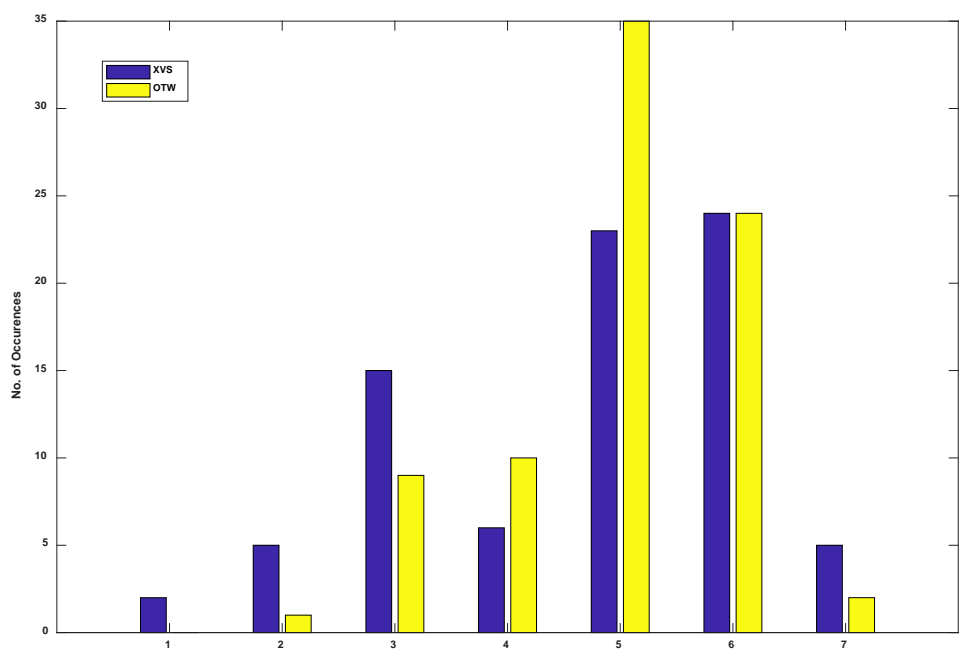

Fig. 14 Ease of Identifying Traffic Aircraft (1: Very Hard; 4: Neutral; 7: Very Easy)

These pilot perceptions and the post-run qualitative data reflect not only the difficulty of the see-and-avoid function, but also the following three factors in the test and the design of the XVS:

1. The pilots were briefed on the intended function of the XVS - a window replacement technology to fill the fuselage obstruction of the LBFD - but the relatively small FOV of the XVS, especially in comparison to the forward-facing windows in the cockpit, still was apparent in the pilots' opinions. For instance, the pilots noted that the "cockpit view had real-time peripheral view that I believe help with tallies". The pilots felt hampered by the small FOV of the XVS saying that the "field of regard/view would need to be expanded" and the "only disadvantage is smaller FOV".

2. The image processing of the XVS imagery was very effective in increasing the contrast; however, the increased contrast also creates a noisier background from which to attempt visual target acquisition. Clouds were most problematic, but "ground clutter made contrasting difficult". We did not allow variation of the image contrast. It was either on or off. Several pilots noted that "environmentals contrast enhancement either helps or hurts. Needs to be automatically adjusted or adjustable". 
3. Lastly, the improved performance for traffic detection of the medium-sized aircraft may have been an artifact of the image processing algorithm and the target aircraft. The medium-sized aircraft - the Falcon jet - is not a clean aircraft. As several pilots noted the "most compelling visual feature was exhaust smoke plume using 'processed' imagery"; "image processing helped seeing exhaust of the Falcon."

The overall conclusion from the vast majority of the subject pilots was that XVS provided comparable performance in the difficult task of seeing and recognizing traffic in challenging see-and-avoid encounters.

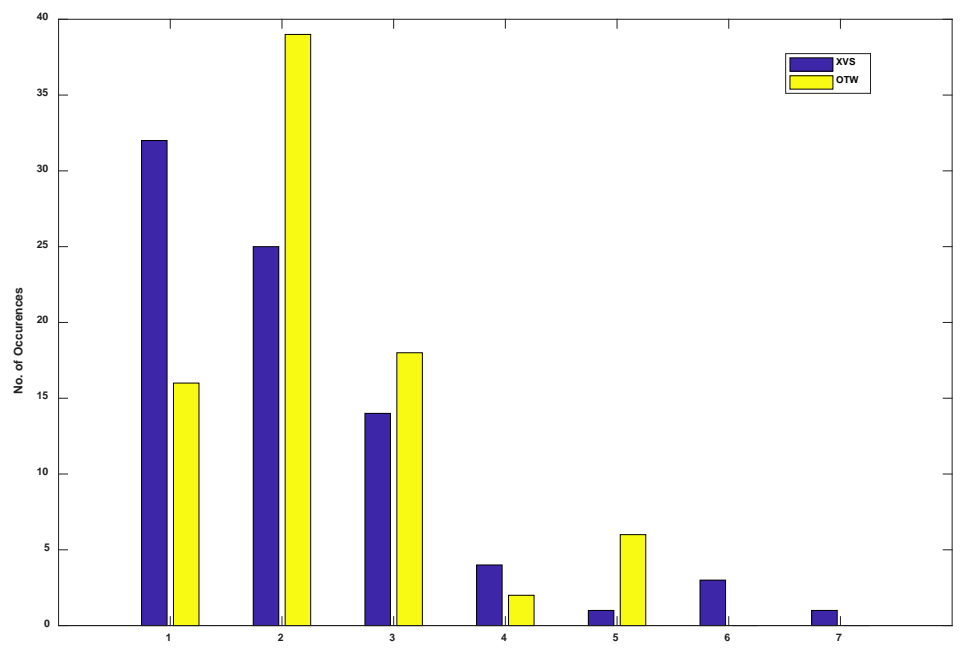

Fig. 15 Sufficient time to assess and react to the traffic? (1: Strongly Agree; 7: Strongly Disagree)

\section{Recommendations}

Based on the data, several recommendations were made for forward work:

- For a better one-to-one comparison, the forward-facing windows should be masked or at least, the FOV of the XVS should be marked on the windows to limit the scan of the OTW pilot to be comparable to the XVS.

- Image processing significantly improved traffic detection, but depending upon the atmospheric conditions, the clouds, and the intrusion geometry, the image processing was felt to possibly hinder detection. As a minimum, the image processing should have an automatic mode with a manual, controllable option available.

- A medium-sized target aircraft other than a "dirty jet" should be used.

- Virginia offers significant atmospheric challenges to visibility - especially haze - but other environmental obscurants should be tested, such as smog and various cloud formations and layers.

\section{Concluding Remarks}

The overall configuration and forward fuselage / nose shaping of the LBFD design are critical to meeting the program goals for sonic boom signature. But this shaping significantly impacts the natural vision for the pilot provided by cockpit windows. Forward-facing windows are not practical for this design.

NASA will design an eXternal Vision System (XVS) that, with other aircraft systems and subsystems, will ensure safe and efficient operations in all phases of flight. XVS is a combination of display, sensor, and computing technologies which create an electronic view, forward of the cockpit, analogous to forward-facing windows.

A flight test was completed assessing a preliminary design for the XVS. The flight test specifically assessed, by direct side-by-side comparison, the performance of a pilot to see and recognize near-collision traffic incursions using either forward-facing windows or an XVS.

The data suggests that the XVS was as good, if not better than forward-facing windows for traffic detection. The data also highlights that image processing to increase the contrast of the video image was a key element of this performance.

The eight subject pilots provided data and commentary assessing the XVS intended function. In particular, the one question for the pilots was whether the preliminary XVS was sufficient for traffic detection/identification/assessment. Three of the pilots noted that:

- "Yes, I was skeptical...but tallies were easy and obvious." 
- "Yes. In some cases it is better."

- "Yes. Being able to adjust brightness and contrast enhances the display unlike the naked eye and windows."

These data suggest confidence that the preliminary XVS design holds promise for meeting the LBFD design challenges. Trade studies will be completed using laboratory and outdoor testing for refinement of the image processing algorithms and for Bayer color format handling, specific to human perception for target acquisition of visual camera information.

\section{Acknowledgements}

The authors would like to express their appreciation and gratitude for the outstanding work by the LaRC Research Services Directorate and the flight test crew - in particular, Taylor Thorson, Rick Yasky, Greg Slover, and David Perez. Their expertise and professionalism in setting up and executing this research was critical to the success of the program. This work could not have been done without them.

\section{References}

[1] Bailey, R.E., Wilz, S.J., and Arthur, J.J. (Trey), “Conceptual Design Standards for eXternal Visibility System (XVS) Sensor and Display Resolution.” NASA Langley Research Center, NASA/TM-2012-217340, Feb 2012.

[2] Australian Transport Safety Bureau. "Limitations of the see-and-avoid principle." April 1991.

[3] Andrews, J.W., "Unalerted Air-to-Air Visual Acquisition.” MIT Report ATC-152, Nov. 1991.

[4] “Contrast-Limited Adaptive Histogram Equalization,” Chapter 8, pp. 474-485, Graphics Gems IV. Zuiderveld, Karel, 1994.

[5] Shelton, K.J., Williams, S.P., Kramer, L.J., Arthur, J.J., Prinzel, L. and Bailey, R.E.. "External Vision Systems (XVS) proofof-concept flight test evaluation.” In SPIE, Degraded Visual Environments: Enhanced, Synthetic, and External Vision Solutions, Vol. 9087, p. 90870F, 2014. 